\title{
Melanoma anorrectal, reporte de un caso y revisión de la literatura
}

\author{
Anorectal melanoma: a case report and literature review \\ Zaira Eunice Montes-Osorio, ${ }^{*}$ José Manuel Moreno-Berber**
}

\begin{abstract}
Palabras clave: Melanoma anorrectal, tratamiento, melanoma maligno, melanoma mucoso, recto.

Key words: Anorectal melanoma, treatment, malignant melanoma, mucosal melanoma, rectum.
\end{abstract}

* Cirugía General y Máster en Administración de Servicios de Salud. ** Cirugía de Colon y Recto, Hospital Ángeles Puebla.

Hospital General Zona Norte, SSEP, Puebla.

Recibido: 03/05/2016 Aceptado: 31/10/2017

\section{RESUMEN}

El melanoma anorrectal es un tumor raro de origen neuroectodérmico que constituye el $1 \%$ de los tumores malignos colorrectales y entre el 0.4 y $1.6 \%$ de los melanomas en general. Habitualmente son lesiones polipoides con o sin pigmentación. En el momento del diagnóstico, el $60 \%$ de los pacientes presentan afectación ganglionar y el $20 \%$ metástasis a distancia. Para su estadificación, se utiliza el sistema simplificado de Ballantyne. Tiene un peor pronóstico en comparación con el melanoma cutáneo. La cirugía es el tratamiento de elección, pero existe una importante controversia acerca de la extensión de la resección. Caso clínico: Masculino de 50 años; inició con dolor y aumento de volumen a nivel perianal, de carácter progresivo. Fue manejado como absceso isquiorrectal y sometido a tratamiento quirúrgico en forma urgente; se identificó una lesión neoplásica de aspecto polipoide dependiente del recto, con extensión hacia la región perianal y absceso agregado. La colonoscopia mostró obstrucción del 90\% de la luz del recto distal, el reporte histopatológico de la resección-biopsia fue compatible con melanoma anorrectal. Se efectuó colostomía laparoscópica y manejo paliativo con radioterapia.

\section{ABSTRACT}

Anorectal melanoma is a rare tumor of neuroectodermal origin that makes up $1 \%$ of colorectal cancers and between 0.4 and $1.6 \%$ of melanomas in general. They are usually polypoid lesions with or without pigmentation. At the time of diagnosis, $60 \%$ of patients have lymph node involvement and $20 \%$ distant metastasis. For staging, the simplified Ballantyne system is used. It has a worse prognosis compared with cutaneous melanoma. Surgery is the treatment of choice, but there is significant controversy about the extent of the resection surgery. Clinical case: 50-year-old male; he presented with progressive pain and volume increase in the perianal region. It was treated as ischiorectal abscess and underwent urgent surgical treatment. A neoplastic lesion of polypoid aspect was identified, dependent on the rectum, extending to the perianal region, as well as an added abscess. The colonoscopy showed $90 \%$ of lumen obstruction of distal rectum; the histopathological report of the tumor resectionbiopsy was compatible with anorectal melanoma. Laparoscopic colostomy and palliative management with radiotherapy were carried out.

\section{INTRODUCCIÓN}

$\mathrm{E}^{\prime}$ melanoma de la región perianal y rectal (MA) es un tumor raro de origen neuroectodérmico que constituye el $1 \%$ de los tumores malignos colorrectales, ${ }^{1,2}$ y entre el 0.4 y $1.6 \%$ de los melanomas en general. ${ }^{3,4}$ Ubicado dentro de los melanomas mucosos, el diagnóstico suele realizarse de forma tardía debido a su localización en zonas de difícil acceso a la exploración y a la falta de signos específicos tempranos. ${ }^{5}$ Más de 76,250 casos de melanoma son diagnosticados cada año en Estados Unidos; sólo el 1\% proviene de los melanocitos de las superficies mucosas, de las cuales la región de cabeza y cuello, mucosa anorrectal y mucosa vulvovaginal son las más afectadas, ${ }^{3,6}$ con una distribución de aproximadamente 55, 24 y $18 \%$, respectivamente. ${ }^{7}$

La incidencia de melanoma rectal es cerca de 2.7 casos por 10 millones de habitantes en forma anual en los Estados Unidos. ${ }^{8,9}$ Sólo el 20 a 30\% de los MA están localizados en el recto; el resto pueden encontrarse en el canal anal o en el margen anal. ${ }^{8}$ El MA es la lesión más común entre los melanomas del tracto gastrointestinal. ${ }^{10}$ Éste ocurre entre los 65-70 años de edad, con predominio en mujeres. . $^{8,9,11,12}$

La mayoría de los pacientes afectados son de raza caucásica (cerca del 95\%). ${ }^{13}$ 
Después del melanoma cutáneo y el ocular, el MA ocupa el tercer lugar en las localizaciones más comunes de esta entidad. ${ }^{12}$

En fechas recientes se ha demostrado la existencia de melanocitos en la zona de transición por debajo de la línea dentada, aumentando en forma distal al anodermo; esto ha llevado a la teoría de que el melanoma se origina desde melanocitos normales en forma distal a la línea dentada, con extensión proximal al recto. ${ }^{14}$

Se sabe que la exposición a rayos ultravioleta, especialmente UV-B con una longitud de onda de 290 a 320 nanómetros (nm), es un claro factor de riesgo para desarrollar melanoma, así como mutaciones en el gen BRAF para melanomas cutáneos, mientras que las mutaciones en el gen c-Kit que promueven la proliferación y propagación celular representan la alteración más común en los melanomas mucosos. ${ }^{15,16}$

Los pacientes comúnmente presentan rectorragia (54-78\%) con o sin dolor anal (14-27\%), y en general son diagnosticados en forma errónea como enfermedad hemorroidal (8-16\%). ${ }^{3,17}$ En algunos otros casos existe fatiga, pérdida de peso, anemia, cambios en el tránsito intestinal u oclusión (6\%) y la presencia de un tumor (12-16\%) en la región inguinal cuando existe metástasis. ${ }^{16,18,19}$ Cabe recordar que el retraso en el diagnóstico deriva de la confusión con lesiones benignas: abscesos, hemorroides y pólipos rectales. ${ }^{10,14,17,18}$

El diagnóstico clínico puede ser incorrecto en el $80 \%$ de los casos, ${ }^{20,21}$ e incluso el histopatológico, en especial en casos de lesiones amelanóticas con características morfológicas inusuales, que pueden ser confundidas con linfoma, carcinoma o sarcoma. ${ }^{22}$ Histológicamente, es característico el pigmento de melanina, aunque es mucho más específica la inmunohistoquímica con positividad para HMB-45 y proteína S-100. ${ }^{23}$

No hay un estándar en cuanto a las guías para un adecuado abordaje diagnóstico de los melanomas mucosos, en específico anorrectales. ${ }^{24}$

Una importante historia clínica y exhaustivo examen físico son importantes para el diagnóstico. En cuanto a la estadificación, debe incluir tacto rectal, colonoscopia, ultrasonido endoscópico, tomografía axial computada, resonancia magnética y tomografía por emisión de positrones (PET-CT), la cual puede ser utilizada para evaluar la profundidad e invasión, metástasis regional y metástasis distal. Notablemente, la resonancia magnética es el método estándar para la evaluación del área de lesión. ${ }^{16}$ La colonoscopia puede revelar lesiones exofíticas oscuras y, en otros casos, amelanóticas, de las cuales se obtendrán biopsias. ${ }^{16}$

En el momento del diagnóstico, el 60\% de los pacientes presentan afectación ganglionar y el $20 \%$ metástasis a distancia. ${ }^{5}$ Para su estadificación, se utiliza el sistema simplificado de Ballantyne $^{25}$ (estadio I: enfermedad localizada, cualquier T NOM0; estadio II: afectación ganglionar regional, TN1M0; estadio III: metástasis distal -cualquier T cualquier N M1), y la American Joint Committee on Cancer se refiere a enfermedad metastásica distal como estadio IV. ${ }^{24,25}$ Se ha evidenciado que la estadificación de acuerdo al tamaño del tumor no correlaciona bien con las tasas de supervivencia, pocos estudios utilizan el nivel de Breslow en melanoma anorrectal; sin embargo, la profundidad de invasión de la lesión parece ser más predictiva de supervivencia en algunas series. ${ }^{1}$ La clasificación de Clark no es de utilidad debido a la naturaleza avanzada del tumor y la ausencia de las capas clásicas de la dermis en la región. 1,25

El tratamiento del MA no ha sido estandarizado debido a la baja incidencia y la falta de evidencia. Por ahora, los resultados sólo son evaluados mediante la tasa de supervivencia de los pacientes afectados. ${ }^{16,19}$ Existen varias modalidades de abordaje para el MA, incluidas la resección, quimioterapia, radioterapia e inmunoterapias; sin embargo, no se ha definido el tratamiento de elección. ${ }^{7,16}$ Cualquier evidencia de enfermedad sistémica contraindica un abordaje agresivo. La resección local está recomendada cuando es técnicamente realizable, debido a que el pronóstico no cambia aun con cirugía radical. ${ }^{1}$ Por lo general, la resección quirúrgica se considera un tratamiento de primera opción para el MA. Tradicionalmente, la resección abdominoperineal es la cirugía estándar para MA, ya que se prefiere por el mayor control de la diseminación linfática y garantiza un margen negativo mayor para el control local. Ishizone y sus colaboradores ${ }^{26}$ reportan que la resección abdominoperineal y la linfadenectomía regional deben efectuarse cuando existe invasión de la submucosa con 
una alta morbilidad y mortalidad. ${ }^{16,26}$ Zhang $^{27}$ evalúa en su estudio el tratamiento quirúrgico de MA en China y compara la supervivencia de los pacientes sometidos a resección abdominoperineal (RAP) contra resección local amplia (RLA) de 1995 a 2007 de un total de 54 pacientes estudiados, 39 con RAP versus 15 con RLA; mediante el test de Kaplan-Meier, reporta que no hay diferencia significativa en cuanto a la supervivencia con ambos procedimientos quirúrgicos ( $p=0.281)$. Finalmente, concluyen que el pronóstico del MA después de la cirugía es muy pobre y los pacientes sometidos a RAP no tienen una ventaja significativa en cuanto a la supervivencia en comparación con aquellos sometidos a RLA. ${ }^{27,28}$

La resección abdominoperineal laparoscópica fue reportada por Ganesh ${ }^{29}$ por primera vez en el 2009 como tratamiento del melanoma anorrectal, con buenos resultados; en la serie de Han y su grupo, ${ }^{30}$ los cuatro pacientes en estadio III recibieron, además, cisplatino e interferón como terapia adyuvante después de la RAP laparoscópica, con una supervivencia de 32 meses. $^{30}$

\section{CASO CLÍNICO}

Masculino de 50 años de edad, sin antecedentes de importancia para su padecimiento, el cual inició hace dos semanas con presencia de dolor moderado y constante, así como aumento de volumen a nivel de la región perianal. A la exploración física de la región perianal, se observó importante proceso inflamatorio, exudado y áreas de necrosis, lesión parduzca de dos centímetros aproximadamente, infiltrativa; fue imposible realizar el tacto rectal (Figura 1).

Fue sometido a exploración y drenaje quirúrgico; se encontró una lesión neoplásica de aspecto melanótico dependiente del recto, con extensión hacia la región perianal, así como áreas con absceso y necrosis. Se realizó drenaje del absceso y resección parcial de la lesión dependiente del recto distal; el espécimen se envió a estudio histopatológico. La colonoscopia con toma de biopsia reveló lesión neoplásica exofítica violácea que se extendía en sentido proximal y obstruía el $90 \%$ de la luz del recto distal; se prolongaba hasta los 15 centímetros del margen anal (Figura 2).

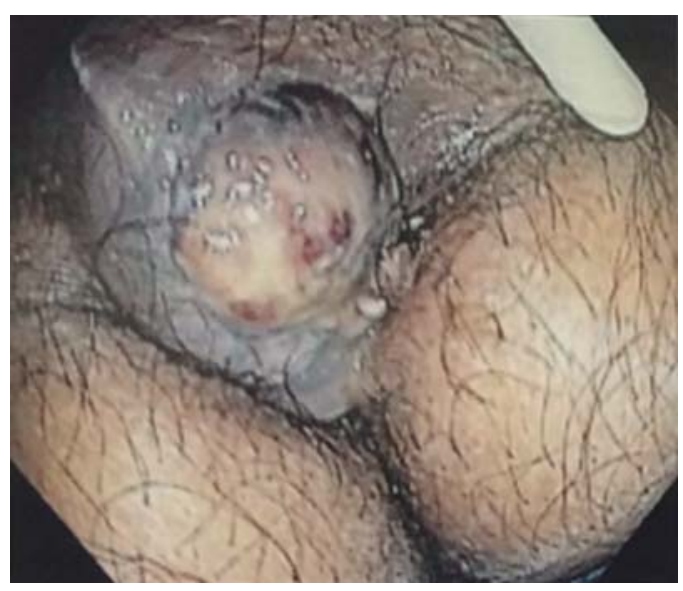

Figura 1. Colonoscopia: lesión perianal.
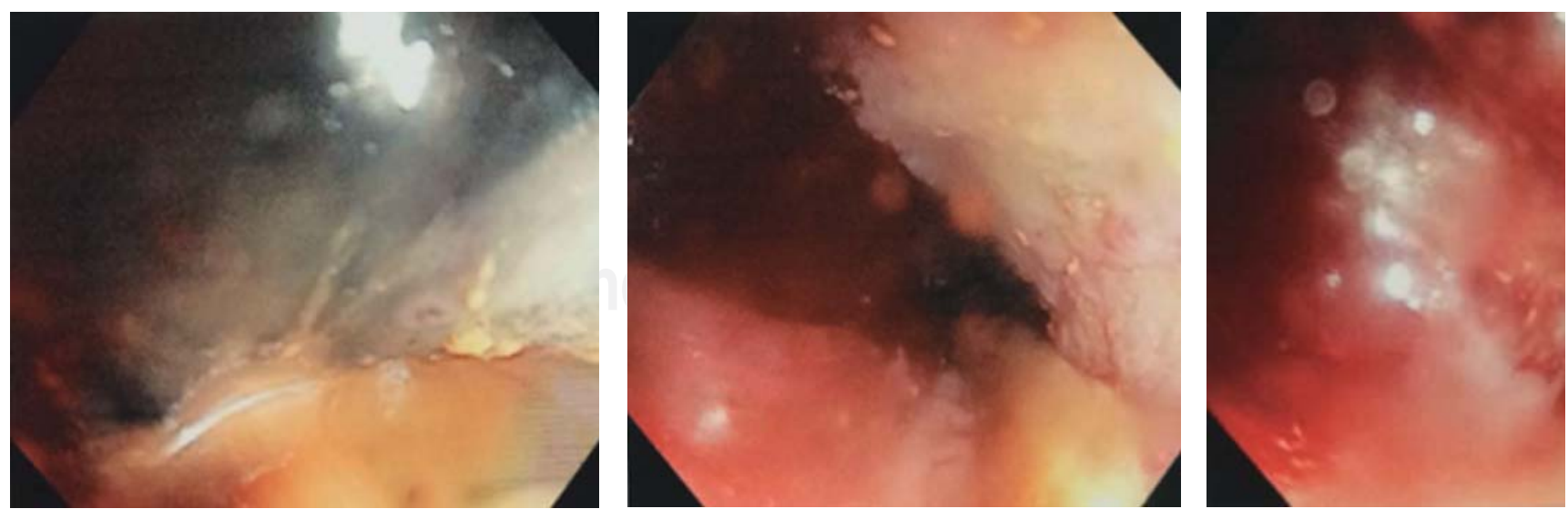

Figura 2. Colonoscopia: lesión recto-proximal, distal y en conducto anal. 

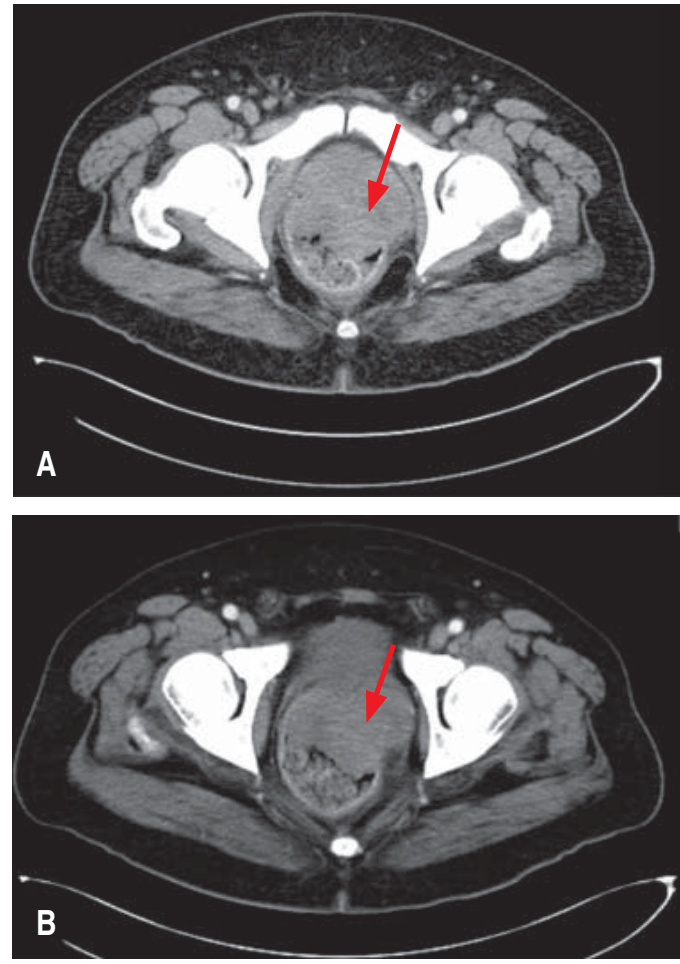

Figuras 3 A y B. Tomografía axial computada contrastada de pelvis, que revela una lesión dependiente del recto de carácter oclusivo e invasivo.

La tomografía axial computada abdominopélvica con contraste mostró lesión dependiente del recto, que lo ocluía en forma total, de carácter invasivo, hasta la región perianal, así como actividad ganglionar a nivel inguinal (Figura 3 A y B).

El análisis del ADN genómico del tumor fue positivo para una mutación V600E del oncogén BRAF. El reporte histopatológico concluyó melanoma con células pequeñas con extensa necrosis, hemorragia e inflamación aguda abscedada. El estudio inmunohistoquímico con anticuerpos monoclonales fue positivo para HMB45 y Melan A del tumor anorrectal. Efectuamos colostomía en asa laparoscópica y envío a oncología médica para tratamiento paliativo con radioterapia (Figura 4).

\section{DISCUSIÓN}

Es claro que aquellos pacientes con enfermedad sistemática al momento del diagnóstico deberán ser tratados conservadoramente, con

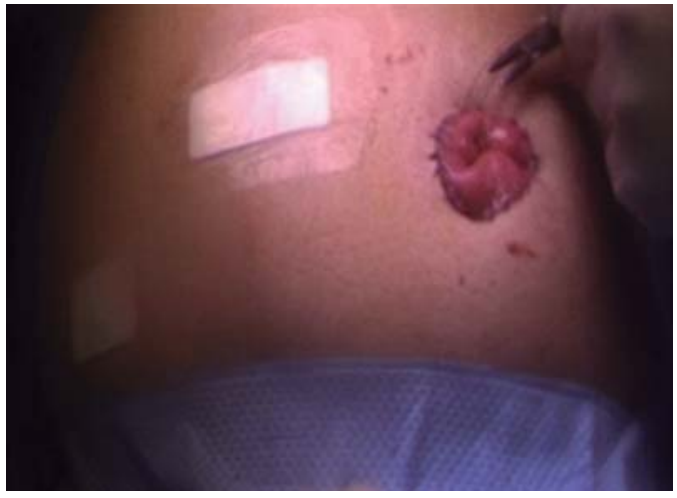

Figura 4. Colostomía en asa realizada por laparoscopia.

escisión local, colostomía o manejo sintomático únicamente. ${ }^{31}$ La serie más grande reportada compara la resección abdominoperineal y la escisión local ampliada en los Estados Unidos mediante la base de datos SEER de 143 pacientes, la cual no reveló diferencias en la supervivencia a cinco años entre ambos métodos quirúrgicos (RAP 16.8 y $19.3 \%$ ELA). ${ }^{30}$ La resección con preservación del esfínter con un margen de uno o dos centímetros se recomienda para tumores con un espesor menor a un milímetro, y entre uno y cuatro milímetros, respectivamente. Si el espesor del tumor es mayor a cuatro milímetros, se recomienda la resección abdominoperineal o la escisión local ampliada en combinación con terapia adyuvante. ${ }^{30}$

En lo que respecta al debate de la alta incidencia de recurrencia local después de la escisión local ampliada, el MD Anderson Cancer Center de la Universidad de Texas ha utilizado desde 1990 la combinación de escisión local con radioterapia. ${ }^{30}$

Aunque la quimioterapia y la inmunoterapia son utilizadas en varias series de casos, su efectividad aún es limitada y se encuentra en debate. $^{26}$

El pronóstico es malo, con una supervivencia a cinco años de entre seis y $22 \%,{ }^{19,28}$ con un rango de ocho a 19 meses. ${ }^{16}$ Debido a su baja incidencia, el melanoma anorrectal del subtipo melanoma mucoso no ha sido muy bien estudiado y casi todas las publicaciones son reportes o serie de casos. La conducta terapéutica es motivo de debate por la insuficiente evidencia que existe. $5,7,28$ 


\section{REFERENCIAS}

1. Martínez-Hernández-Magro P, Villanueva-Sáenz E, Chávez-Colunga LB. Melanoma maligno anal. Reporte de casos y revisión de la literatura. Rev Gastroenterol Mex. 2009; 74: 39-44.

2. Frank W, Kurban RS, Hoover HC Jr, Sober AJ. Anorectal melanoma. A case report and brief review of the literature. J Dermatol Surg Oncol. 1992; 18: 333-336.

3. Turner G, Abbott S, Eglinton T, Wakeman C, Frizelle F. Anorectal melanoma: not a haemorrhoid. N Z Med J. 2014; 127: 73-81.

4. Van Schaik PM, Ernst MF, Meijer HA, Bosscha K. Melanoma of the rectum: a rare entity. World J Gastroenterol. 2008; 14: 1633-1635.

5. Ballester-Sánchez R, Unamuno-Bustos B, Navarro-Mira M, Botella-Estrada R. Actualización en melanoma mucoso. Actas Dermosifiliogr. 2015; 106: 96-103.

6. Postow MA, Hamid O, Carvajal RD. Mucosal melanoma: pathogenesis, clinical behavior, and management. Curr Oncol Rep. 2012; 14: 441-448.

7. Carcoforo P, Raiji MT, Palini GM, Pedriali M, Maestroni U, Soliani G, et al. Primary anorectal melanoma: an update. J Cancer. 2012; 3: 449-453.

8. Chen H, Cai Y, Liu Y, He J, Hu Y, Xiao Q, et al. Incidence, surgical treatment, and prognosis of anorectal melanoma from 1973 to 2011: a populationbased SEER analysis. Medicine (Baltimore). 2016; 95: e2770.

9. Coté TR, Sobin LH. Primary melanomas of the esophagus and anorectum: epidemiologic comparison with melanoma of the skin. Melanoma Res. 2009; 19: 58-60.

10. Mihajlovic M, Vlajkovic S, Jovanovic P, Stefanovic V. Primary mucosal melanomas: a comprehensive review. Int J Clin Exp Pathol. 2012; 5: 739-753.

11. Tomicic J, Wanebo HJ. Mucosal melanomas. Surg Clin North Am. 2003; 83: 237-252.

12. McLaughlin CC, Wu XC, Jemal A, Martin HJ, Roche $\mathrm{LM}$, Chen VW. Incidence of noncutaneous melanomas in the U.S. Cancer. 2005; 103: 1000-1007.

13. Cheung MC, Perez EA, Molina MA, Jin X, Gutierrez $J C$, Franceschi D, et al. Defining the role of surgery for primary gastrointestinal tract melanoma. J Gastrointest Surg. 2008; 12: 731-738.

14. González QH, Bahena-Aponte JA, Martínez-Benítez B. Melanoma amelanótico anorrectal. Rev Gastroenterol Mex. 2011; 76: 171-172.

15. Webster JD, Kiupel M, Yuzbasiyan-Gurkan V. Evaluation of the kinase domain of c-KIT in canine cutaneous mast cell tumors. BMC Cancer. 2006; 6: 85.

16. Guo J, Qin S, Liang J, Lin T, Si L, Chen X, et al. Chinese guidelines on the diagnosis and treatment of melanoma (2015 Edition). Ann Transl Med. 2015; 3: 322.

17. Trzcinski R, Kujawski R, Mik M, Sygut A, Dziki L, Dziki A. Malignant melanoma of the anorectum -a rare entity. Langenbecks Arch Surg. 2010; 395: 757-760.

18. Buissin D, Sterle A, Schmiegelow P, Wassenberg D, Ambe PC. Primary anorectal malignant melanoma: a rare but aggressive tumor: report of a case. World J Surg Oncol. 2015; 13: 12.
19. Nam S, Kim CW, Baek SJ, Hur H, Min BS, Baik SH, et al. The clinical features and optimal treatment of anorectal malignant melanoma. Ann Surg Treat Res. 2014; 87: 113-117.

20. Das G, Gupta S, Shukla PJ, Jagannath P. Anorectal melanoma: a large clinicopathologic study from India. Int Surg. 2003; 88: 21-24.

21. Gavriilidis P, Moula E, Nikolaidou A. Primary rectal malignant melanoma-case report. Hippokratia. 2013; 17: 380-381.

22. Banerjee SS, Harris M. Morphological and immunophenotypic variations in malignant melanoma. Histopathology. 2000; 36: 387-402.

23. Díaz-Sánchez A, Lara MA, Ortega P, Aramendi T, González C, Alberdi JM, et al. Melanoma colorrectal: revisión de dos formas diferentes de presentación. Gastroenterol Hepatol. 2011; 34: 83-88.

24. Seetharamu N, Ott PA, Pavlick AC. Mucosal melanomas: a case-based review of the literature. Oncologist. 2010; 15: 772-781.

25. Vidrio-Morgado H. Estudio comparativo entre dos sistemas de estadificación para el melanoma de mucosas en cabeza y cuello. México, División de Estudios de Postgrado e Investigación. Instituto Nacional de Cancerología. Universidad Nacional Autónoma de México. 15-4-0016. 2013. 5-8.

26. Ishizone S, Koide N, Karasawa F, Akita N, Muranaka F, Uhara $\mathrm{H}$, et al. Surgical treatment for anorectal malignant melanoma: report of five cases and review of 79 Japanese cases. Int J Colorectal Dis. 2008; 23: 1257-1262.

27. Zhang S, Gao F, Wan D. Abdominoperineal resection or local excision? a survival analysis of anorectal malignant melanoma with surgical management. Melanoma Res. 2010; 20: 338-341.

28. Matsuda A, Miyashita M, Matsumoto S, Takahashi G, Matsutani T, Yamada T, et al. Abdominoperineal resection provides better local control but equivalent overall survival to local excision of anorectal malignant melanoma: a systematic review. Ann Surg. 2015; 261 : 670-677.

29. Ramalingam G, Gan EY, Kutt-Sing W. Laparoscopic abdominoperineal resection for anorectal melanoma: a case report and review of the literature. Surg Laparosc Endosc Percutan Tech. 2009; 19: e149-e151.

30. Han J, Shi C, Dong X, Wang J, Wen H, Wang B, et al. Laparoscopic abdomino-perineal resection for patients with anorectal malignant melanoma: a report of 4 cases. J Biomed Res. 2016; 30: 436-440.

31. Terán-Porcayo MA, Gómez del Castillo-Rangel AC. Melanoma anorrectal: Informe de once pacientes. Cir Gen. 2003; 25: 223-227.

Correspondencia:

Zaira Eunice Montes-Osorio

Departamento de Cirugía General, Hospital General Zona Norte, Servicios de Salud del Estado de Puebla. 88 Poniente y 7 Norte, Infonavit San Pedro, 72230, Puebla, México.

Teléfono: 22-28-37-89-66

E-mail: drazairaeunice@gmail.com 